Supporting Information for the Manuscript:

\title{
Electrostatically Assembled Multilayered Films of Biopolymer Enhanced Nanocapsules for On-Demand Drug Release
}

Li Xu, ${ }^{*}{ }^{\dagger}$ Zihan Chu, ${ }^{\dagger}$ Hailong Wang, ${ }^{\dagger}$ Lawrence Cai, ${ }^{\dagger}$ Zhigang Tu, ${ }^{\dagger}$ Hanqing Liu, ${ }^{\dagger}$ Chunyin Zhu, ${ }^{\dagger}$ Haifeng Shi, ${ }^{*} \dagger$ Donghui Pan,, Jia Pan $^{\S}$ Xiang Fei ${ }^{\dagger}$

$\dagger$ Institute of Life Sciences, Jiangsu University, Zhenjiang, Jiangsu 212013, China

Jjiangsu Institute of Nuclear Medicine, Wuxi, Jiangsu 214063, China

§Novo Nordisk Research Center - Indianapolis, Inc., Indianapolis, IN 46241, USA

Email: 1xu66@ujs.edu.cn, shihf@ujs.edu.cn
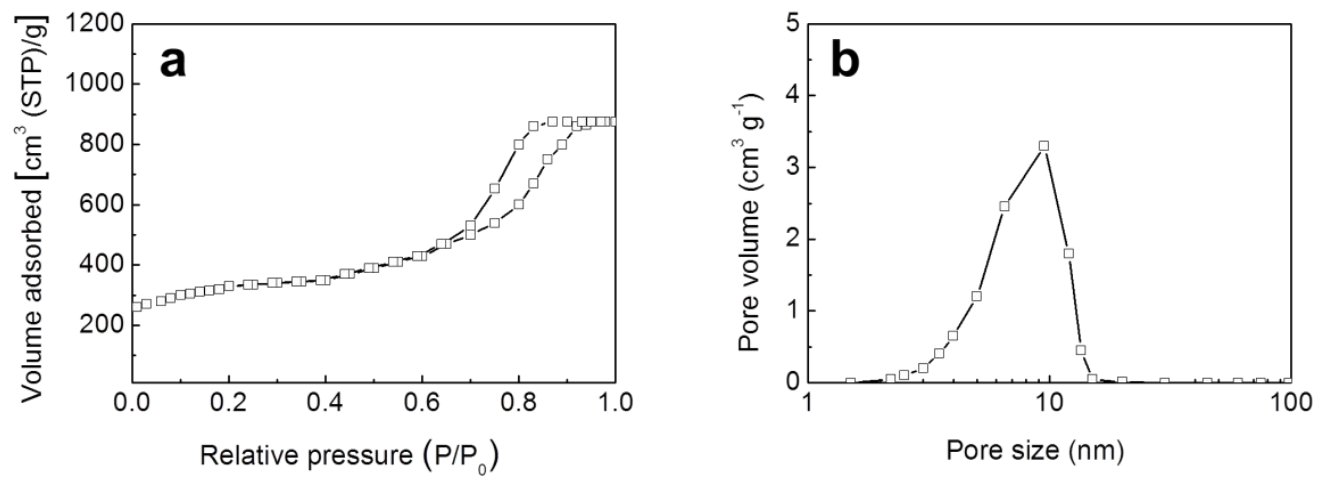

Figure S1. (a) Nitrogen adsorption-desorption isotherm and (b) BET of SNC materials.

Table S1. Properties from Brunauer-Emmett-Teller (BET) analysis of SNC materials.

\begin{tabular}{cc}
\hline Properties & SNC materials \\
\hline $\mathrm{S}_{\mathrm{BET}}\left(\mathrm{m}^{2} \mathrm{~g}^{-1}\right)$ & 355 \\
$\mathrm{~V}_{\mathrm{BET}}\left(\mathrm{cm}^{3} \mathrm{~g}^{-1}\right)$ & 0.91 \\
Pore size $(\AA)$ & 93.6 \\
\hline
\end{tabular}



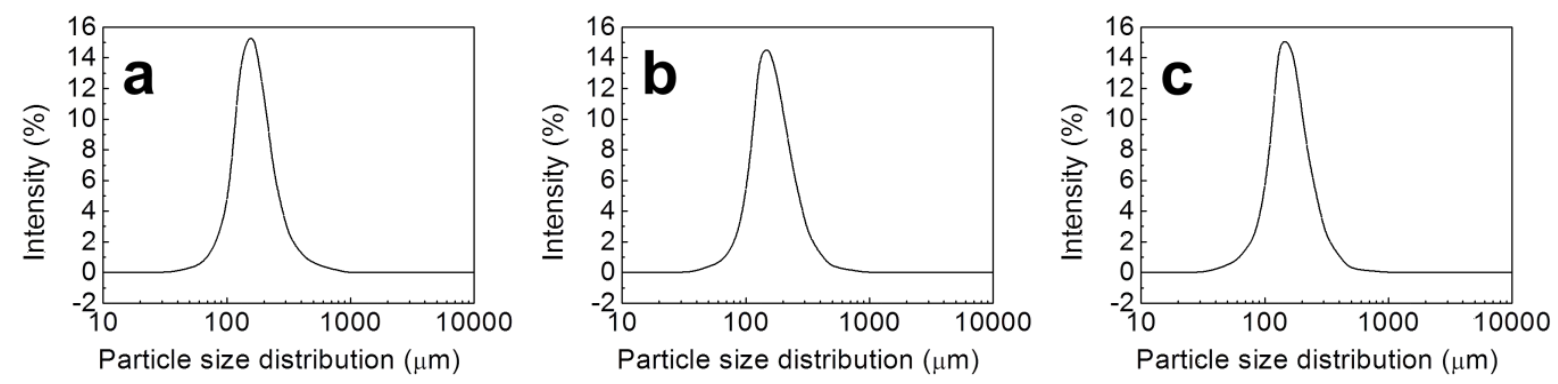

Figure S2. Hydrodynamic particle size of bare SNCs in $0.01 \mathrm{M}$ phosphate buffer solutions at pH (a) 5.0, (b) 5.5 and (c) 7.4, respectively, measured by dynamic light scattering (DLS) at $25^{\circ} \mathrm{C}$. Prior to DLS measurement, the solutions were kept for 24 hours to reach the equilibrium.
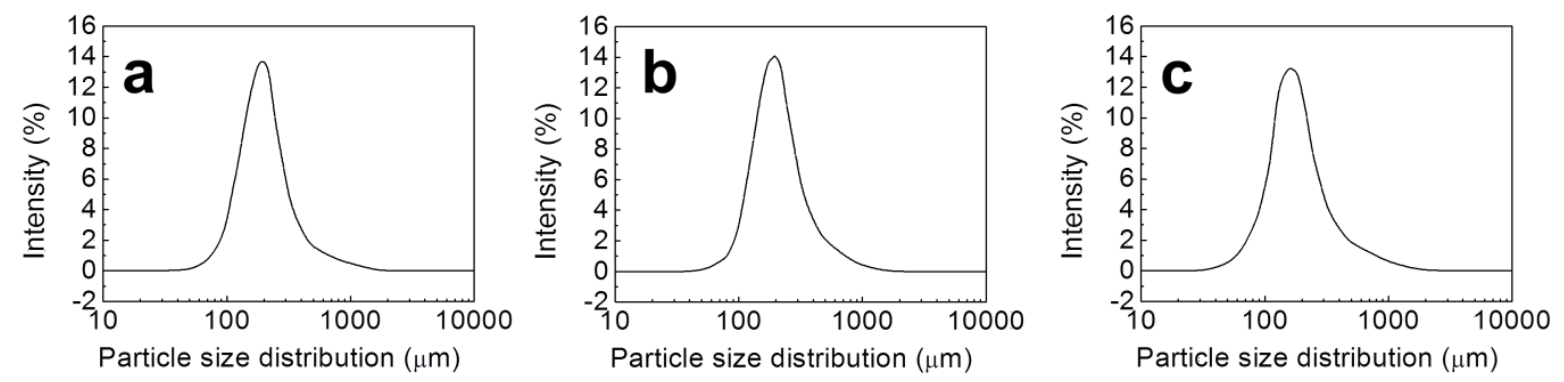

Figure S3. Hydrodynamic particle size of biopolymer coated SNCs in $0.01 \mathrm{M}$ phosphate buffer solutions at $\mathrm{pH}$ (a) 5.0, (b) 5.5 and (c) 7.4, respectively, measured by DLS at $25^{\circ} \mathrm{C}$. Prior to DLS measurement, the solutions were kept for 24 hours to reach the equilibrium. 


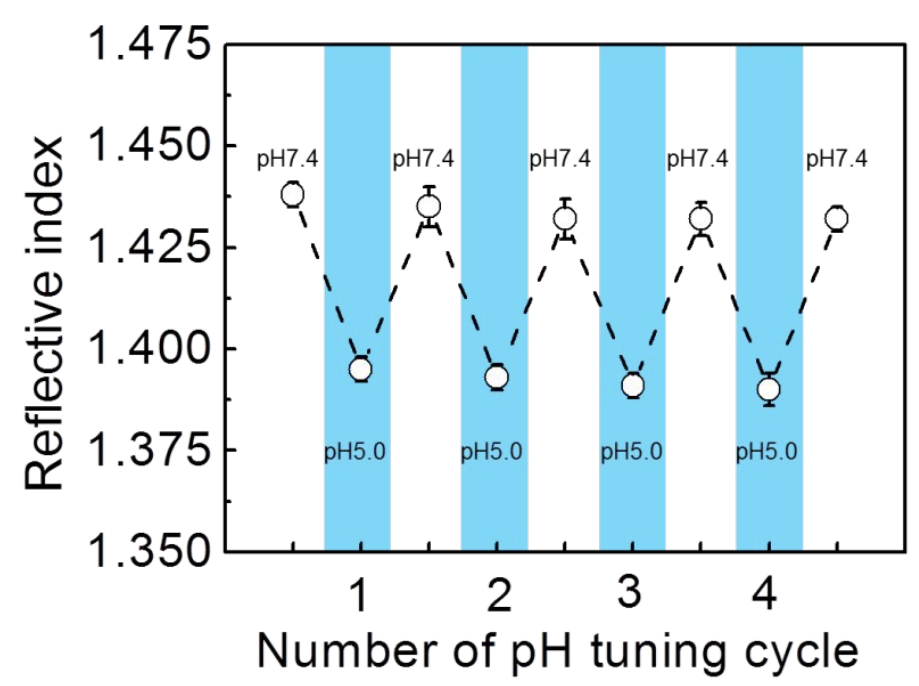

Figure S4. Reflective index during reversible $\mathrm{pH}$-induced swelling of $[\mathrm{SNC} / \mathrm{CS}]_{3}$ films in $0.01 \mathrm{M}$ phosphate buffer solutions at pH 7.4 and 5.0, respectively, measured by in situ ellipsometry with alternating 5-min film incubations in $37^{\circ} \mathrm{C}$ solutions.
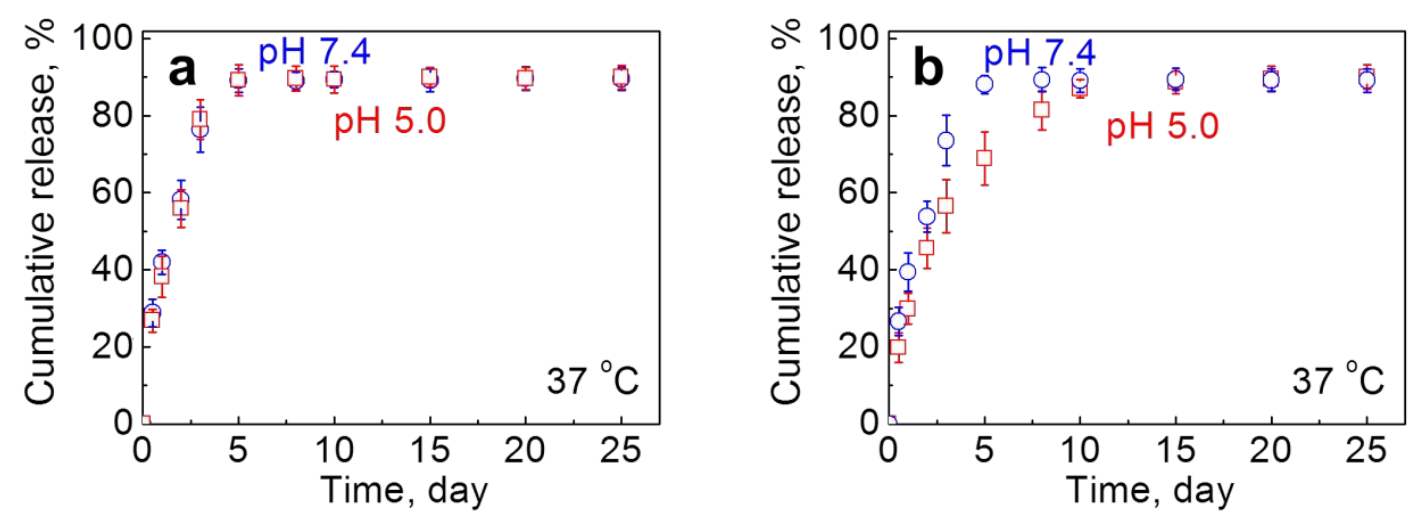

Figure S5. Release kinetics of fulvestrant from free standing (a) bare SNCs and (b) biopolymercoated SNCs in $20 \mathrm{~mL}$ of $37^{\circ} \mathrm{C}$ buffer solution with $\mathrm{pH}$ values of 7.4 (circles) and 5.0 (squares), respectively. Fulvestrant release was monitored by measuring fulvestrant accumulated in PBS solution by HPLC/MS. 

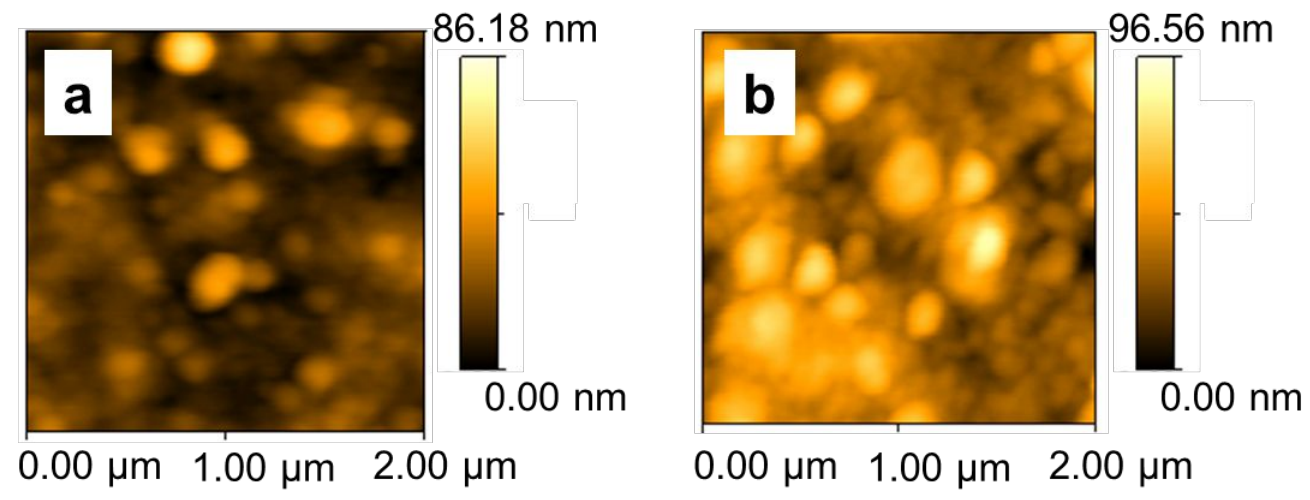

Figure S6. AFM topography images of $[\mathrm{SNC} / \mathrm{CS}]_{3}$ films after being immersed in PBS with $\mathrm{pH}$ values of (a) 7.4 , and (b) 5.0 at $37^{\circ} \mathrm{C}$ for 120 days, respectively.

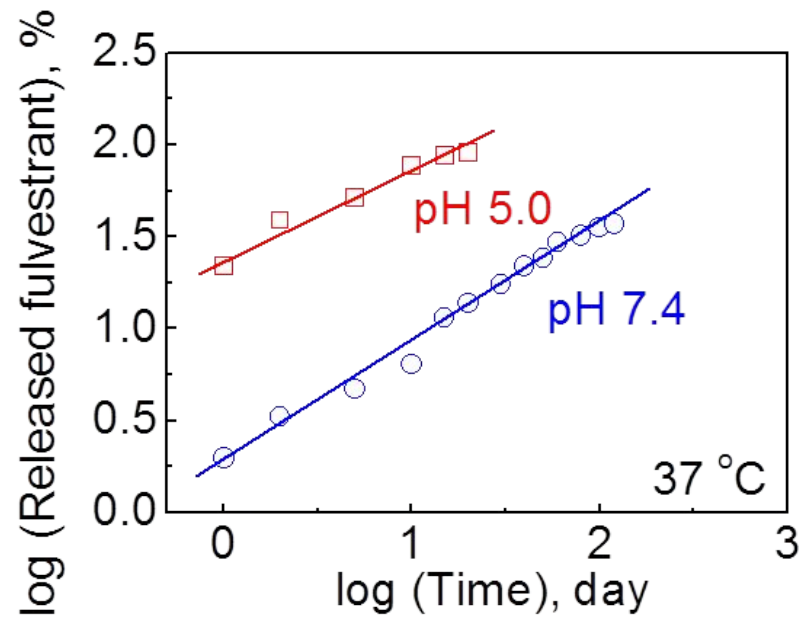

Figure S7. Log-log plot of kinetics of fulvestrant release from $[\mathrm{SNC} / \mathrm{CS}]_{3}$ films at $\mathrm{pH} 7.4$ (circles) and 5.0 (squares), respectively. Fulvestrant release was conducted in PBS at $37^{\circ} \mathrm{C}$. 